\title{
Trivium
}

Revue franco-allemande de sciences humaines et sociales - Deutsch-französische Zeitschrift für Geistesund Sozialwissenschaften

1 | 2008

"Iconic Turn » et réflexion sociétale

\section{En ordre dispersé}

Introduction

\section{Georges Didi-Huberman}

\section{(2) OpenEdition}

Journals

Édition électronique

URL : http://journals.openedition.org/trivium/351

DOI : $10.4000 /$ trivium.351

ISSN : 1963-1820

\section{Éditeur}

Les éditions de la Maison des sciences de l'Homme

\section{Référence électronique}

Georges Didi-Huberman, « En ordre dispersé », Trivium [En ligne], 1 | 2008, mis en ligne le 09 avril 2008, consulté le 22 septembre 2020. URL : http://journals.openedition.org/trivium/351 ; DOI : https:// doi.org/10.4000/trivium.351

Ce document a été généré automatiquement le 22 septembre 2020.

\section{(c) $(1) \&$}

Les contenus des la revue Trivium sont mis à disposition selon les termes de la Licence Creative Commons Attribution - Pas d'Utilisation Commerciale - Pas de Modification 4.0 International. 


\title{
En ordre dispersé
}

\author{
Introduction
}

\author{
Georges Didi-Huberman
}

1 Je me souviens d'une réflexion que me fit, il y a une quinzaine d'année, Stephen Bann lors d'un de ses passages à Paris : il venait visiter celles et ceux - de Julia Kristeva à Jean Louis Schefer ou de Jean-Claude Lebensztejn à Hubert Damisch - qui, à ses yeux, constituaient ce que la dite French Thought pouvait offrir comme contribution à la plus récente pensée des images. Cette réflexion était esquissée sous la forme d'un trait d'humour, comme toujours chez Stephen Bann: les Français, les Parisiens avant tout, sont - ou bien ont tendance à se comporter comme - des stars, c'est-à-dire, disait-il, des étoiles très, très éloignées les unes des autres. Ces étoiles forment-elles une constellation? Toute la question est là. Elle demeure, aujourd'hui encore, irrésolue, offusquée par la complexité des jeux d'influences entre les étoiles fixes et leurs satellites, les nébuleuses et leurs champs magnétiques, les forces de gravitation et les passages météoriques, les décompositions d'énergie sidérale et les apparitions de novae (les étoiles nouvelles)...

2 Il devrait revenir à nos amis étrangers - et non à moi, qui me sens si peu Français en France, si French (donc misunderstood) aux USA et si « continental» dans une Allemagne intellectuelle beaucoup plus en travail et en dialogue que partout ailleurs - de décider si les étoiles en question forment une constellation et, si oui, quel aspect revêt finalement celle-ci. Je ne peux, dans le cadre de ce début d'échange qu'incarne ici le projet Trivium, que me référer à quelques impressions issues d'une expérience fatalement partielle, quoique déjà longue. La première chose frappante est le rôle négatif joué, grosso modo, par les institutions universitaires françaises. Dans le domaine de l'histoire de l'art, l'Université - je parle d'un temps dominé par les disciples directs d'André Chastel - a longtemps barré la route à tout ce qui pouvait se faire d'intéressant dans le domaine de la réflexion sur les images. Les personnalités originales se trouvaient alors poussées dans les marges, soit du côté des écoles d'art, soit du côté de l'École des hautes Études en Sciences sociales, soit dans d'inattendus départements des facultés (la littérature comparée, par exemple, où l'on retrouve un penseur des images tel que Bernard 
Vouilloux) ou du CNRS (où quelqu'un comme Marie-José Mondzain a quelque peu erré, semble-t-il).

3 Voilà pourquoi les quatre textes choisis dans ce premier ensemble sont dûs à des chercheurs que l'université n'accueille - presque - pas : Philippe-Alain Michaud a dirigé une revue de cinéma expérimental, programmé les films du Louvre et dirige actuellement les collections cinématographiques du Musée national d'Art moderne; Didier Semin a quitté le Centre Pompidou pour l'École nationale supérieure des BeauxArts ; Jean-Pierre Criqui travaille pour la Délégation aux Arts plastiques et dirige Les Cahiers du Musée national d'Art moderne; seul le plus jeune, Bertrand Prévost - signe des temps, espérons-le -, parvient depuis peu à faire son bout de chemin dans le grand méandre académique.

4 Cette situation globalement négative est due, sans doute, au compartimentage disciplinaire extrêmement rigide en France. Il est difficile - même à l'EHESS, où l'on y peut perdre sa possibilité d'habilitation - de travailler un jour sur Fra Angelico et, quelques années plus tard, sur Georges Bataille ou l'art contemporain. Les petites étoiles ont donc tendance à s'éloigner des grands magmas institutionnels. Voilà pourquoi la pensée de l'image, en France, procède par ordre dispersé. Voilà pourquoi, bien qu'il soit intéressant de lire Jacques Rancière et Henri Maldiney, il apparaît assez clairement que Rancière n'a jamais lu Maldiney et que Maldiney ne lira jamais Rancière. Les quelques exemples de travail collectif se trouvent - signe encourageant, à nouveau - chez des chercheurs plus jeunes qui ont décidé d'explorer leur champ d'étude sans pour autant s'affilier unilatéralement à une prise de position théorique donnée d'avance (je pense, en particulier, au travail mené par la Société française de Photographie et à sa revue Études photographiques).

Il y a, dans cette dispersion, une considérable déperdition de possibilités. Un manque criant de dialogue. Une ignorance qui s'installe de part et d'autre de frontières souvent minuscules. La situation éditoriale contribue dramatiquement à cette situation puisque, à côté des grands éditeurs " généraux » qui publient au compte-goutte leurs essais sur l'art, une structure comme celle de L'Harmattan ne possède ni la qualité édotoriale, ni la diffusion d'un éditeur tel que Wilhelm Fink en Allemagne. Il y a pourtant un aspect positif et fécond à cette dispersion même. Quelque chose qui offre un remarquable champ de possibilités, en dépit de tous les obstacles. En se dispersant, les étoiles nous montrent, mieux que dans tout système solaire - c'est-à-dire centralisé -, combien vaste est l'espace que l'on peut traverser, que l'on peut explorer. En cela elle déploie le champ, ouvre de nouveaux horizons, rend possible l'apparition des comètes les plus inattendues.

6 Cette situation doit être analysée dans l'économie du discours sur l'art en tant que genre littéraire. La France, de ce point de vue, hérite d'une tradition considérable, celle qui, dans le champ poétique, a voulu rompre dès le XVIII ${ }^{\mathrm{e}}$ siècle avec le discours normatif des académies. Cela va de Diderot à Jean Genet, de Baudelaire à Francis Ponge, de Mallarmé à Yves Bonnefoy, etc. Les textes théoriques les plus marquants sur l'image, en France, portent presque tous, en conséquence, la marque de ce profond ancrage poétique : c'est Georges Bataille écrivant sur Lascaux, Maurice Blanchot sur « Les deux versions de l'imaginaire", Michel Foucault sur les Ménines, Roland Barthes sur la photographie. Même la tradition plus strictement philosophique - je pense aux écrits de Gilles Deleuze sur Francis Bacon, le cinéma ou la théorie de l'image selon Beckett; ou bien à ceux de Jacques Derrida sur le sublime, l'aveuglement, la dimension tactile - 
adopte la liberté des poètes pour malmener, devant les images, toute pensée esthétique qui chercherait un peu trop son propre mode de définition stricto sensu.

7 C'est sans doute pourquoi les grands mots d'ordre théoriques qui, dans le monde anglosaxon ou allemand, réussissent à créer une véritable effusion globale, se trouvent constamment détournés ou, mieux, modulés. Ainsi, le fameux iconic turn n'apparaît pas en France comme une réelle «nouveauté » dans la mesure où les modèles mêmes de la nouveauté théorique, dans la pensée française, ont presque toujours suivi les chemins du détour ou du retour: un travail de relecture plutôt qu'une annonce de pure nouveauté. C'est Foucault relisant Binswanger, Derrida relisant Husserl et Heidegger, Lacan relisant Freud, Deleuze relisant Spinoza... L'iconic turn prend alors la forme d'un return qui aura produit la relecture sémiotique d'Erwin Panofsky par la génération structuraliste (Pierre Bourdieu, Hubert Damisch, Louis Marin), et qui produit désormais une relecture philosophique d'Aby Warburg, à la fois comme penseur des survivances (le passé inaperçu de notre histoire de l'art) et penseur de l'actualité (le présent inaperçu de notre culture des images). Comme autrefois Baudelaire avait rouvert le champ littéraire en traduisant Poe, comme Lacan aura réinventé le champ psychanalytique tout entier en relisant les mots de Freud, il est à souhaiter, dans le meilleur des cas, que cette relecture non philologique de Warburg - comme, par ailleurs, celle de Walter Benjamin, d'Alois Riegl ou de Konrad Fiedler, pour ne nommer que ceux-là - puisse aider à réinterpréter, à réinventer, à rouvrir l'histoire et la pensée des images.

\section{BIBLIOGRAPHIE}

Barthes, Roland, Mythologies, Paris, Le Seuil, 1957 (rééd. 1970).

Barthes, Roland, La Chambre claire. Note sur la photographie, Paris, Le Seuil / Cahiers du Cinéma / Gallimard, 1980.

Barthes, Roland, L'Empire des signes (1970), Paris, Flammarion, 1980.

Barthes, Roland, Essais critiques, vol. III : L'obvie et l'obtus, Paris, Le Seuil, 1982.

Bataille, Georges, Lascaux ou la naissance de l'art, Genève, Skira, 1955.

Bataille, Georges, Les Larmes d'Éros, Paris, Pauvert, 1961 (rééd. 2001).

Blanchot, Maurice, La Part du feu, Paris, Gallimard, 1949.

Blanchot, Maurice, L’Espace littéraire, Paris, Gallimard, 1955 (rééd. 1996).

Blanchot, Maurice, L'Entretien infini, Paris, Gallimard, 1969.

Blanchot, Maurice, Le Pas au-delà, Paris, Gallimard, 1973.

Blanchot, Maurice, La Bête de Lascaux, Montpellier, Fata Morgana, 1982.

Criqui, Jean-Pierre, The Tempo of Painting. Brice Marden: Attendants, Bears, and Rocks, New York, Matthew Marks Gallery, 2002. 
Criqui, Jean-Pierre, Un trou dans la vie. Essais sur l'art depuis 1960, Paris, Desclée de Brouwer, 2002. Criqui, Jean-Pierre, Wim Delvoye. Sept propos sur le sexe, les rayons X et les cochons, Genève, Galerie Guy Bärtschi, 2002.

Criqui, Jean-Pierre (dir.), Robert Morris. Blind Time Drawings, 1973-2000, Prato / Göttingen, Centro per l'Arte contemporaneo Luigi Pecci / Steidl, 2005.

Damisch, Hubert, Théorie du nuage. Pour une histoire de la peinture, Paris, Le Seuil, 1972.

Damisch, Hubert, Fenêtre jaune cadmium, ou les dessous de la peinture, Paris, Le Seuil, 1984.

Damisch, Hubert, L'Origine de la perspective, Paris, Flammarion, 1987.

Damisch, Hubert, Le Jugement de Pâris. Iconographie analytique, I, Paris, Flammarion, 1992.

Damisch, Hubert, Traité du trait, Paris, RMN, 1995.

Damisch, Hubert, La Dénivelée. À l'épreuve de la photographie, Paris, Le Seuil, 2001.

Deleuze, Gilles, Francis Bacon. Logique de la sensation, Paris, La Différence, 1981.

Deleuze, Gilles, Cinéma, 1. L’image-mouvement, Paris, Minuit, 1983.

Deleuze, Gilles, Cinéma, 2. L'image-temps, Paris, Minuit, 1985.

Deleuze, Gilles, Le Pli. Leibniz et le baroque, Paris, Minuit, 1988.

Deleuze, Gilles, Critique et clinique, Paris, Minuit, 1993.

Deleuze, Gilles, L'T̂le déserte et autres textes. Textes et entretiens, 1953-1974, éd. D. Lapoujade, Paris, Minuit, 2002.

Deleuze, Gilles, Deux Régimes de fous. Textes et entretiens, 1975-1995, éd. D. Lapoujade, Paris, Minuit, 2003.

Derrida, Jacques, La Vérité en peinture, Paris, Flammarion, 1978.

Derrida, Jacques, Mémoire d'aveugle. L'autoportrait et autres ruines, Paris, RMN / Musée du Louvre, 1990.

Derrida, Jacques, Le Toucher, Jean-Luc Nancy, Paris, Galilée, 2000.

Foucault, Michel, Les Mots et les choses. Une archéologie des sciences humaines, Paris, Gallimard, 1966. Foucault, Michel, La Pensée du dehors, Montpellier, Fata Morgana, 1986.

Foucault, Michel, La Peinture de Manet (1971), Paris, Le Seuil, 2004.

Kristeva, Julia, Polylogue, Paris, Le Seuil, 1977.

Kristeva, Julia, Visions capitales, Paris, RMN, 1998.

Lebensztein, Jean-Claude, Zigzag, Paris, Flammarion, 1981.

Lebensztein, Jean-Claude, Chahut, Paris, Hazan, 1989.

Lebensztein, Jean-Claude, L'Art de la tache. Introduction à la « Nouvelle Méthode » d'Alexander Cozens, Paris, Éditions du Limon, 1990.

Lebensztein, Jean-Claude, Annexes - de l'œuvre d'art, Bruxelles, La Part de l'œiil, 1999.

Maldiney, Henri, Regard, parole, espace, Lausanne, L'Âge d'Homme, 1973.

Maldiney, Henri, L'Art, l'éclair de l'être, Paris, Comp'Act, 1993.

Maldiney, Henri, Ouvrir le rien. L'art nu, La Versanne, Encre marine, 2000. 
Marin, Louis, Études sémiologiques. Écritures, peintures, Paris, Klincksieck, 1971.

Marin, Louis, Détruire la peinture, Paris, Galilée, 1977.

Marin, Louis, Le Portrait du roi, Paris, Minuit, 1981.

Marin, Louis, Des pouvoirs de l'image. Gloses, Paris, Le Seuil, 1993.

Marin, Louis, De la représentation, Paris, Gallimard / Le Seuil, 1994.

Marin, Louis, Philippe de Champaigne ou la présence cachée, Paris, Hazan, 1995.

Marin, Louis, Sublime Poussin, Paris, Le Seuil, 1995.

Marin, Louis, Opacité de la peinture. Essais sur la représentation au Quattrocento (1989), Paris, Éditions de l'EHESS, 2006.

Michaud, Philippe-Alain, Aby Warburg et l'image en mouvement, Paris, Macula, 1998.

Michaud, Philippe-Alain, Le Peuple des images. Essai d'anthropologie figurative, Paris, Desclée de Brouwer, 2002.

Michaud, Philippe-Alain, (dir.), L'Horreur comique. Esthétique du Slapstick, Centre Pompidou, 2004.

Michaud, Philippe-Alain, (dir.), Comme le rêve le dessin, Paris, Éditions du Centre Pompidou / Éditions du Louvre, 2005.

Michaud, Philippe-Alain, (dir.), Le Mouvement des images, Paris, Éditions du Centre Pompidou, 2006.

Michaud, Philippe-Alain, Sketches. Histoire de l'art, cinéma, Paris, Kargo / L'Éclat, 2006.

Mondzain, Marie-José, Image, icône, économie. Les sources byzantines de l'imaginaire contemporain, Paris, Le Seuil, 1996.

Mondzain, Marie-José, L'Image peut-elle tuer?, Paris, Bayard, 2002.

Mondzain, Marie-José, Le Commerce des regards, Paris, Le Seuil, 2003.

Mondzain, Marie-José, Homo spectator, Paris, Bayard, 2007.

Prévost, Bertrand, La Peinture en actes. Gestes et manières dans l'Italie de la Renaissance, Arles, Actes Sud, 2007.

Rancière, Jacques, Le Partage du sensible. Esthétique et politique, Paris, La Fabrique, 2000.

Rancière, Jacques, La Fable cinématographique, Paris, Le Seuil, 2001.

Rancière, Jacques, L'Inconscient esthétique, Paris, Galilée, 2001.

Rancière, Jacques, Le Destin des images, Paris, La Fabrique, 2003.

Rancière, Jacques, Malaise dans l'esthétique, Paris, Galilée, 2004.

Semin, Didier, Le Peintre et son modèle déposé, Genève, Mamco, 2001.

Semin, Didier, La Piste du hérisson, Nîmes, Jacqueline Chambon, 2004.

Semin, Didier, Le Sablier de Penone, via Piero della Francesca, Alfred Hitchcock et Andreï Tarkovski, Paris, L'Échoppe, 2005.

Semin, Didier, Grenouillages. Brisset, Goya, Lavater, Paris, L'Échoppe, 2006.

Vouilloux, Bernard, L'Interstice figural. Discours, histoire, peinture, Sainte-Foy (Québec) / Grenoble, Le Griffon d'argile / PUG, 1994. 
Vouilloux, Bernard, La Peinture dans le texte. XVIIIe-XXe siècles, Paris, Éditions du CNRS, 1994.

Vouilloux, Bernard, Le Tableau vivant. Phryné, l'orateur et le peintre, Paris, Flammarion, 2002.

Vouilloux, Bernard, L'Euvre en souffrance. Entre poétique et esthétique, Paris, Belin, 2004.

INDEX

Schlüsselwörter : Iconic turn, Theorien zum Bild

Mots-clés : iconic turn, pensée des images

\section{AUTEUR}

\section{GEORGES DIDI-HUBERMAN}

Maître de conférence, École des hautes études en sciences sociales, Paris 